\title{
Duodenal fat intensifies the perception of heartburn
}

\author{
J H Meyer, A Lembo, J D Elashoff, R Fass, E A Mayer
}

Department of Medicine, Division of Gastroenterology at the West Los Angeles Healthcare Center of the VA Greater Los Angeles Healthcare System, UCLA School of Medicine, Los Angeles, CA, USA J H Meyer

UCLA/CURE Neuroenteric Disease Program, UCLA School of Medicine, Los Angeles, CA, USA A Lembo

R Fass

E A Mayer

Biostatistics Core Research Institute at the Cedars Sinai Medical Center, UCLA School of Medicine, Los Angeles, CA, USA J D Elashoff

Correspondence to: Dr E A Mayer, Rm 223, Bldg 115, CURE, West Los Angeles VAMC, 11301 Wilshire Boulevard, Los Angeles, CA 90073, USA. emayer@ucla.edu

Accepted for publication 5 December 2000

\begin{abstract}
Background-Patients with gastrooesophageal reflux disease (GORD) frequently report that meals high in fat worsen heartburn. Nevertheless, studies to determine whether high fat meals promote gastro-oesophageal reflux have produced conflicting and equivocal conclusions.
\end{abstract}

Patients and methods-To determine, alternatively, whether fat in the small intestinal lumen intensifies the perception of heartburn, we studied 11 patients with typical heartburn from GORD. After being placed on omeprazole to suppress endogenous acid, these fasting subjects underwent oesophageal perfusions with graded doses of $\mathrm{HCl}$ at $\mathrm{pH}$ values of 1.0 , $1.5,2.0$, and 2.5. Oesophageal perfusions were conducted while the duodenum was perfused with saline (control) and again with fat at $8 \mathrm{~g} / \mathrm{h}$.

Results-Time to onset, intensity, and severity of heartburn varied with dose of oesophageal acid $(p<0.01)$. Time to onset was significantly $(p<0.01)$ shorter, and intensity and severity of heartburn significantly $(p<0.05)$ greater, during duodenal perfusion with fat.

Conclusion-We conclude that duodenal fat intensifies the perception of heartburn.

(Gut 2001;49:624-628)

Keywords: gastro-oesophageal reflux disease; heartburn; perception; fat

Patients commonly report that foods high in fat aggravate heartburn ${ }^{1}$ but the basis for this association is poorly understood. While ingested or intestinally perfused fat lowers resting pressures of the lower oesophageal sphincter (LOS) in normal subjects, ${ }^{23}$ the degree of lowering is insufficient to account for an increased reflux of gastric contents into the oesophagus. More recently, other investigators ${ }^{4-9}$ have sought to determine whether high (versus low) fat meals increase the frequency of transient relaxations of the LOS and the frequency of oesophageal reflux, but the results of these investigations have been conflicting and generally have not demonstrated that high fat meals promote reflux.

Fat in the intestinal lumen evokes a variety of motor and secretory responses that are not perceived but there is increasing recognition that luminal fat also alters conscious perceptions: it triggers feelings of fullness (satiety), intensifies vection induced motion sickness, and alters thresholds for and quality of perceptions of gastric distensions. ${ }^{10-13}$ Experimental observations indicate ${ }^{14} 15$ that nutrients trigger facilitatory pathways for pain by activating a subset of vagal afferents that enhance visceral sensitivity. Therefore, we postulated that fat in the gastrointestinal lumen may intensify the perception of heartburn by lowering the threshold to pain during acidification of the oesophagus.

We determined in subjects who suffered from frequent heartburn whether the time to onset, intensity, and severity of heartburn were altered during controlled perfusions of acid into the oesophagus while the duodenum was simultaneously perfused with fat versus saline treatment alone (in control tests).

\section{Methods}

We studied 11 patient volunteers (nine men, two women; mean age 48 (range 28-74) years) who complained of daily typical heartburn. Patients were excluded if they had a history of comorbid conditions (such as coronary artery disease, peptic ulcer disease, Barrett's oesophagus, or gastric surgery) of if they were receiving medications known to affect the gastrointestinal tract. Eight of the 11 patients had previously undergone a 24 hour ambulatory oesophageal $\mathrm{pH}$ study which confirmed abnormal oesophageal reflux (mean per cent of time below pH 4 was $9.4 \%$; range $6.5-18 \%$ ); two of these individuals had endoscopically determined erosive oesophagitis and a ninth subject had been shown to have erosive oesophagitis but had never been tested with 24 hour $\mathrm{pH}$ probes. The remaining two subjects had long histories of classic heartburn that had been completely relieved by daily omeprazole. Four of the subjects had grade 1 oesophagitis (Savary-Miller classification) at the pretest endoscopy while the other seven had normal appearing oesophageal mucosa (grade 0 ).

All subjects gave informed consent and the study was approved by the human studies committee at the West Los Angeles VA Medical Center.

\section{STUDY DESIGN}

Our objective was to determine latency to onset and intensity of heartburn during controlled standardised acidifications of the oesophagus with $\mathrm{HCl}$. A previous study ${ }^{16}$ had established that heartburn varied in a dose related fashion during acidifications with diluted $\mathrm{HCl}$ over the

Abbreviations used in this paper: GORD, gastro-oesophageal reflux disease; LOS, lower oesophageal sphincter. 
$\mathrm{pH}$ range 1.0-3.0 (that is, over a concentration of $\mathrm{HCl}$ of $0.1-0.001 \mathrm{M}$ ). Therefore, we studied perceptions of heartburn during five minute infusions of $\mathrm{HCl}$ at $\mathrm{pH} 1.0,1.5,2.0$, and 2.5. To determine how intraduodenal fat might affect dose responsive heartburn, we simultaneously perfused the duodenum at $40 \mathrm{ml} / \mathrm{h}$ with emulsified fat (20\% Intralipid) versus saline (control). The speed of gastric emptying (or duodenal entry) of dietary fat varies with the amount of fat ingested. ${ }^{17}$ Our selected duodenal load of fat $(8 \mathrm{~g} / \mathrm{h})$ was less than the maximal first hour rate $(12 \mathrm{~g} / \mathrm{h})$ of gastric emptying of dietary oil previously observed in normal subjects and approximated the first hour rate of duodenal entry of fat after a meal which might contain $40 \mathrm{~g}$ of fat. ${ }^{17}{ }^{18}$ To minimise reflux of endogenous gastric acid into the oesophagus and thus to ensure that oesophageal acidification was controlled by our perfusions, we gave all subjects omeprazole $20 \mathrm{mg}$ twice daily for two days prior to the test.

\section{STUDY PROTOCOI}

After an overnight fast, all subjects underwent upper gastrointestinal endoscopy on the day before the test so that we could inspect the oesophagus, stomach, and duodenum. Oesophageal mucosal changes were rated (using the Savary-Miller classification), and after the inspections, an Enteroflex 8F feeding tube was passed with endoscopic guidance through the nose, oesophagus, and stomach into the second portion of the duodenum. The tube was then anchored in place by taping its proximal portion to the nose. Following endoscopy, patients were monitored overnight in hospital and were again fasted from midnight. On the next day, we used fluoroscopy to adjust and/or verify the position of the tip of the Enteroflex tube in the second portion of the duodenum.

The next day, subjects were studied while seated upright in a chair. Infusion reservoirs and pumps were placed behind the subjects and solutions were infused through opacified tubes so that subjects were unaware of what solutions they were receiving. Thirty minutes prior to the testing, we started perfusing the duodenum via the Enteroflex tube at a rate of $40 \mathrm{ml} / \mathrm{h}$ with either $0.075 \mathrm{M}$ saline (control) or $20 \%$ Intralipid. Half of the patients received saline first and the other half received Intralipid first. After 30 minutes of perfusion, we began to infuse doses of $\mathrm{HCl}$ via a separate nasal oesophageal tube positioned with its tip at the mid oesophagus. We infused four doses of $\mathrm{HCl}$ (corresponding to $\mathrm{pH}$ values of $1.0,1.5,2.0$, and 2.5) at a rate of $7 \mathrm{ml} / \mathrm{min}$. Each dose was infused for five minutes and was followed by a five minute period of no infusion before the next dose was given. The four doses of acid were administered in random order. If symptoms persisted for three minutes or more after discontinuing the previous dose, $5 \mathrm{ml}$ of water were infused every two minutes until symptoms resolved before the next dose of acid was given.

After completion of the initial oesophageal perfusions, subjects were given a 90 minute rest period during which oesophageal and duodenal perfusions were stopped and subjects were allowed to ambulate ad libitum. At the end of the 90 minute rest period, duodenal perfusion was again started (with saline, if the earlier perfusion had been fat; with fat, if the earlier perfusion had been saline) and 30 minutes later the subject was again tested with the four doses of oesophageal $\mathrm{HCl}$, exactly as previously (in the same order in which the subject had received them earlier). Subjects could not observe and did not know whether they were receiving duodenal saline or Intralipid.

STUDY ASSESSMENTS

Responses to acidification were quantified by three parameters previously utilised by oth$\mathrm{ers}^{19}$ : time to onset of heartburn, intensity of heartburn, and overall severity (intensity $\times$ duration). Time to onset was the time (in seconds) to initial perception of typical heartburn. Subjects were instructed to push a button as soon as they noted heartburn, and the pushed button recorded the time of onset on a computer. For subsequent statistical analyses, one second was added to the $300 \mathrm{sec}-$ onds of acid perfusion if the subject did not experience heartburn during the 300 seconds of exposure to acid. Intensity of typical heartburn was determined with a visual analogue scale. Subjects were instructed to rate the intensity of heartburn at the end of the 300 second period of acid perfusion by marking the line on the scale $(0 \mathrm{~mm}$, no heartburn; $20 \mathrm{~mm}$, extremely intense heartburn). Severity was calculated as the product of duration $\times$ intensity. Duration was determined as 300 seconds minus seconds to onset. Thus severity $=$ duration $\times$ intensity at the end of 300 seconds of acid perfusion. Subjects were also asked to indicate the presence or absence of other gastrointestinal symptoms from a list which included nausea, bloating, fullness, gas, pressure, regurgitation, chest discomfort, and chest pain.

STATISTICAL ANALYSES

The data were bimodal, with seven of the 11 subjects being very sensitive to perfused acid and the other four being much less sensitive. Two methods were used to assess these non-parametric data.

Times to onset were ranked (for example, shortest time to onset was given a rank of 1 ; longest time, a rank of 8) across all eight perfusions (that is, at the four $\mathrm{pH}$ values each during fat and saline duodenal perfusions). If times to onset were the same for two (or more) perfusions, they were given a fractional rank (for example, if two perfusions had the same but second shortest onset, they were each ranked equally at 2.5; the next shortest onset was given a rank score of 4.0). A one group repeated measures ANOVA was then performed on these rank scores and tested for a fat effect and a $\mathrm{pH}$ effect. Two of the 11 subjects (see below) did not complete the oesophageal perfusions at $\mathrm{pH} 1.0$ during either duodenal fat or saline instillations. These missing values were filled in with rank scores of 1.5. 
In the second method, ln transformations were used to determine whether the doseresponse during duodenal fat differed from the dose-response during duodenal saline. In each subject, scores across all four $\mathrm{pH}$ values during duodenal fat were summed, as were the corresponding scores during duodenal perfusions with saline. The $\ln$ of individual sums (fat $v$ saline) were then compared in each subject by paired $t$ tests. Times to onset were computed as 301 seconds in this second method if subjects experienced no heartburn during the $300 \mathrm{sec}-$ onds of observation.

\section{Results}

Oesophageal perfusions at all four $\mathrm{pH}$ values during both duodenal fat and duodenal saline were completed in nine of the 11 subjects. The other two subjects developed severe heartburn and nausea during their initial oesophageal perfusion at $\mathrm{pH} 1$ and therefore the first test at $\mathrm{pH} 1$ was terminated. Neither subject would allow the second perfusion at $\mathrm{pH} 1$ but both completed the other six oesophageal perfusions. Of a total of 86 oesophageal perfusions, 15 were associated with nausea
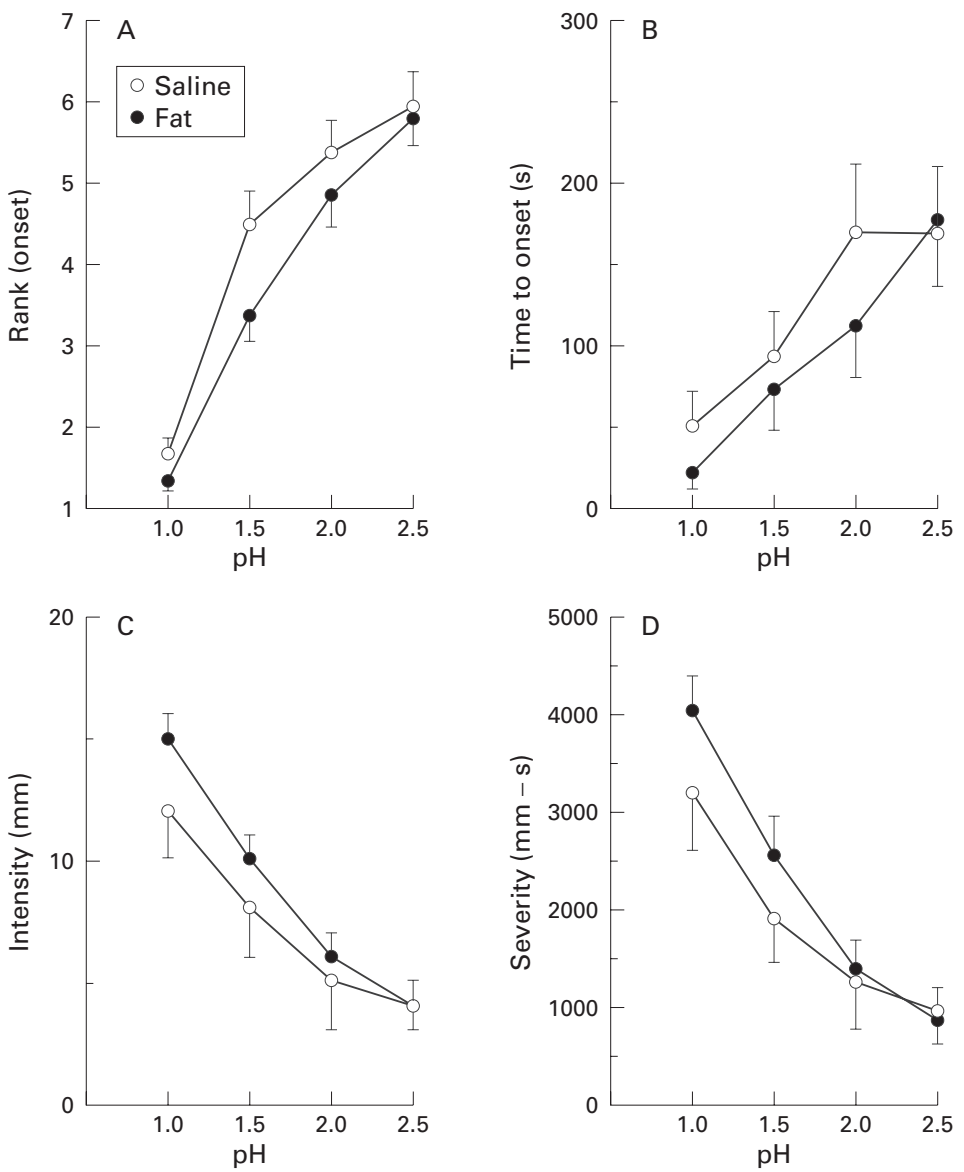

Figure 1 (A) Rank orders (mean (SEM) on a scale of 1-8) for times of onset of heartburn in the 11 subjects during duodenal perfusions with fat versus saline while the oesophagus was perfused with HCl at each of the four $p H$ values $(1.0,1.5,2.0$, and 2.5). Ranks of 1.5 were assigned for oesophageal perfusions at $p H 1.0$ that were missing in two of the subjects (see text). (B-D) Mean (SEM) values for the 11 subjects during duodenal perfusions with fat versus saline while the oesophagus was perfused with $\mathrm{HCl}$ at each of four $p H$ values. There are missing data for two of the 11 subjects when the oesophagus was perfused at pH 1.0 during duodenal instillations of fat and saline. (B) Time (seconds) to onset of heartburn (limits 0-301 seconds). (C) Perceived intensity of heartburn (mm on an analogue scale; limits $0-20 \mathrm{~mm}$ ). (D) Severity of heartburn (duration $\times$ intensity; limits $0-6000 \mathrm{~mm}$-second). (including the terminated tests in the two above subjects). All but four of these 15 episodes of nausea were encountered during oesophageal perfusions at a $\mathrm{pH}$ value 1.0 or 1.5. Seven of the 15 episodes of nausea arose during duodenal instillations of saline

As previously observed, ${ }^{16}$ there was dose related heartburn as infused $\mathrm{HCl}$ was varied from $\mathrm{pH} 1.0$ to $\mathrm{pH} 2.5$ (fig 1 ). Repeated measures ANOVA showed a significant $(\mathrm{p}<0.01)$ $\mathrm{pH}$ effect, with significant linear, quadratic, and cubic correlations between time of onset and oesophageal infusion $\mathrm{pH}$. Both the intensity and severity of heartburn also varied with $\mathrm{pH}$ (fig 1).

Repeated measures ANOVA confirmed that duodenal fat $(v$ saline $)$ significantly $(p<0.01)$ shortened the time to onset of heartburn (fig 1A). Differences in time to onset were significant at the $<0.01$ level whether the analysis was confined to the nine subjects who completed all tests or whether the analyses included the two other subjects with filled in rank scores of 1.5 for the missing data at oesophageal $\mathrm{pH} 1.0$.

Similarly (fig 1B), a paired $t$ test of the $\ln$ transformed sums of onsets across the four $\mathrm{pH}$ values confirmed a significantly $(\mathrm{p}<0.01)$ shorter time to onset for the four oesophageal $\mathrm{pH}$ values when the duodenum was perfused with fat ( $v$ saline) in the nine subjects who completed all tests. Both intensity (fig 1C) and severity (fig 1D) of heartburn were significantly $(p<0.05)$ greater during duodenal fat than during duodenal perfusion with saline in the nine subjects who completed the full protocol.

\section{Discussion}

How fatty meals intensify heartburn in subjects who suffer from pyrosis is poorly understood. Authors of six studies ${ }^{4-9}$ have pursued the hypothesis that high dietary fat may increase the frequency of transitory relaxations of the LOS and/or gastro-oesophageal reflux but despite these multiple attempts, this idea has not been established. ${ }^{20}$ Two of the studies ${ }^{5}$ were flawed by poor designs. Castiglione and colleagues $^{5}$ found more oesophageal reflux in normal subjects after a very high fat $(72 \mathrm{~g})$ meal than after a moderately high fat (32 g) meal but because the high fat meal also contained chocolate that was not present in the lower fat meal, was much more caloric, and because they studied reflux at a different time of day and during longer recumbency after the high fat meal, the results cannot be attributed solely to the effects of high fat. Iwakiri and colleagues ${ }^{6}$ found more oesophageal reflux in recumbent (but not upright) normal subjects after a high fat $(70 \%)$ versus low fat $(16 \%)$ liquid meal but again this observation was confounded by differences in caloric contents of the two meals and by failure to randomise the test sequences. The well designed study of Becker and colleagues ${ }^{4}$ did not have these flaws but the results were peculiar: subjects with frequent heartburn had more gastro-oesophageal reflux than control subjects but no increase in reflux after a high fat $(40 \mathrm{~g})$ meal (compared with an isocaloric low fat $(11 \mathrm{~g})$ meal) whether 
recumbent or upright whereas in asymptomatic control subjects the high fat meal increased reflux when subjects were upright but not when they were recumbent. Penagini and colleagues ${ }^{8}$ found that a high fat $(44 \mathrm{~g})$ meal did not evoke more reflux than a low fat $(20 \mathrm{~g})$ meal in either subjects with heartburn or asymptomatic controls. Similarly, Pehl and colleagues $^{9}$ failed to observe more reflux in asymptomatic subjects after a high fat $(50 \mathrm{~g})$ meal compared with a low fat $(10 \mathrm{~g})$ meal. Holloway and colleagues ${ }^{7}$ observed slightly more gastro-oesophageal reflux in GORD patients (but not asymptomatic controls) in the right lateral decubitus position when the duodenum was perfused with $10 \%$ Intralipid ( $v$ saline) at a duodenal load of $6 \mathrm{~g} / \mathrm{h}$ of fat.

The failure of Paginini et al and Pehl et al to confirm fat induced reflux, the large standard errors in several of the studies, the poor designs in some, and the inconsistent pattern of Becker's findings (fat induced reflux only in normal subjects) led Pehl and colleagues ${ }^{9}$ to conclude that the few positive findings among these reports were the result of type II errors. Rejecting the reflux hypothesis (because of inconsistent results), Peganini et al, on the other hand, considered the alternative that fat in the small intestine might enhance the perception of heartburn without actually intensifying the exciting stimulus of acid reflux. ${ }^{8}$

Until now, the alternative hypothesis has never been tested but our results strongly support this idea. Under the uniform stimuli of controlled acidifications of the oesophagus across the range of dose-responses, physiological loads of duodenal fat shortened the latency to onset of typical heartburn and increased the intensity and severity of heartburn in subjects who had GORD. The load of duodenal fat that we selected was less than the maximal loads that normally enter the duodenum during the first hour of a high fat meal ${ }^{17}$ and would be about equal to loads entering in the first hour after a meal that might contain $40 \mathrm{~g}$ of fat. ${ }^{17}{ }^{18}$ Our subjects experienced nausea about as often during duodenal perfusion with saline as during perfusions with fat, and under either condition nausea was more common during oesophageal perfusions with $\mathrm{HCl}$ at lower $\mathrm{pH}$ values. These observations suggest that nausea was related to oesophageal infusion of $\mathrm{HCl}$ and not duodenal infusions of fat. Thus worsening of perceived heartburn by duodenal fat appeared to be a physiological effect of a fat load that was otherwise well tolerated.

Fat in the intestinal lumen is increasingly recognised to alter sensations that originate from the gastrointestinal tract. ${ }^{10-13}$ Fat enhances the perception of fullness (satiety), a response that originates largely at the level of the gut wall. ${ }^{10}{ }^{21-23}$ Intraduodenal fat intensifies the feeling of nausea associated with vection induced motion sickness. ${ }^{11}$ Duodenally perfused fat also altered the discomfort threshold during gastric distensions with a balloon and changed the quality of sensation. ${ }^{12}{ }^{13}$ In addition to these effects on visceral sensation, ingestion of fat has been shown to decrease sensitivity to cutaneous pain but this last effect does not appear to depend on stimulation of sensors in the gut wall. ${ }^{24}$

The small intestine is innervated with afferent nerves that discharge when fatty acids are present in the intestinal lumen..$^{25}$ Subsets of vagal afferent fibres have been implicated in the activation of both pain facilitating pathways and pain inhibitory pathways ${ }^{13}{ }^{14}$ and recent observations implicate coeliac vagal afferents as part of a tonic pain inhibitory system. The effect of duodenal fat on the perception of fullness or nausea during gastric distensions appears to be mediated by serotonergic ${ }^{26}$ and peptidergic nerves which bear cholesystokinin A receptors ${ }^{27}$ but whether similar nervous pathways are involved in the intensification of heartburn by duodenal fat is unknown. It also remains to be determined whether intraduodenal fat activates the pain facilitatory pathways or deactivates the pain inhibitory pathways to intensify heartburn.

In summary, we found that duodenally infused fat significantly shortened latency to onset of heartburn and intensified the perception of acid induced heartburn in patients with GORD. These findings are consistent with a variety of recent observations that intraluminal nutrients modulate sensitivities of perceptions from the upper gastrointestinal tract.

This study was supported by funds from AstraZeneca R\&D, Sweden, and was carried out in the facilities of the Department of Veterans Affairs Medical Center, West Los Angeles, California, USA.

1 Nebel OT, Fornes MF, Castell DO. Symptomatic gastroesophageal reflux: Incidence and precipitating factors. $A m \mathcal{F}$ Dig Dis 1976;21:953-6.

2 Nebel OT, Castell DO. Lower esophageal pressure changes after food ingestion. Gastroenterology 1972;63:778-83.

3 Nebel OT, Castell DO. Inhibition of the lower oesphageal sphincter by fat - a mechanism of fatty food intolerance. Gut 1973;14:270-4

4 Becker DJ, Sinclair J, Castell DO, et al. A comparison of high and low fat meals on postprandial esophageal acid exposure. Am F Gastroenterol 1989;84:782-6.

5 Castiglione F, Emde C, Armstrong D, et al. Oesphageal pH-metry: Should meals be standardized. Scand $\mathcal{F}$ Gastroenterol 1992;27:350-4

6 Iwakiri K, Konayahsi M, Kotoyori M, et al. Relationship between postprandial esophageal acid exposure and meal volume and fat content. Dig Dis Sci 1996;41:926-30.

7 Holloway RH, Lyrenas E, Ireland A, et al. Effect of Holloway RH, Lyrenas E, Ireland A, et al. Effect of
intraduodenal fat on lower oesophaeal sphincter function intraduodenal fat on lower oesophaeal sphincter functi

and gastro-oesophageal reflux. Gut 1997;40:449-53.
8 Penagini R, Mangano M, Bianchi PA. Effect of increasing the fat content but not the energy load of a meal on gastrooesophageal reflux and lower oesophageal motor function. Gut 1998;42:330-3

9 Pehl C, Waisenhoefer A, Wendl B, et al. Effect of low and high fat meals on lower esophageal motility and gastroesophageal reflux in healthy subjects. $A m \mathcal{F}$ Gastroenterol 1999;94:1192-6.

10 Welch I McL, Sepple CP, Read NW. Comparisons of the effects on satiety and eating behavior of infusion of lipid into the different regions of the small intestine. Gut 1988;29:306-11.

11 Feinle C, Grundy D, Read NW. Fat increases vectioninduced nausea independent of changes in gastric emptyinduced nausea independent of change

12 Feinle C, Grundy D, Read NW. Effects of duodenal nutrients on sensory and motor responses of the human stomach to distension. Am f Physiol 1997;273:G721-6.

13 Barbera R, Feinle C, Read NW. Nutrient-specific modulation of gastric mechanosensitivity in patients with functional dyspepsia. Dig Dis Sci 1995;40:1636-41.

14 Randich A, Gebhart GF. Vagal afferent modulation of nociception. Brain Res Rev 1992;17:77-99.

15 Janig W, Khasar SG, Levine JD, et al. The role of vagal visceral afferents in the control of nociception. In: Mayer EA, Saper CB, eds. The biological basis for mind body interactions. Amsterdam: Elsevier Sciences, 2000:273-87.

16 Price SF, Smithson KW, Castell DO. Food sensitivity in reflux esophagitis. Gastroenterology 1978;75:240-3.

17 Meyer JH, Hlinka M, Kao D, et al. Gastric emptying of oil from solid and liquid meals: Effect of human pancreatic insufficiency. Dig Dis Sci 1996;41:1691-9. 
18 Meyer JH, Elashoff JD, Lake R. Gastric emptying of indigestible $\mathrm{v}$. digestible oils and solid fats in norma human. Dig Dis Sci 1999; 44:1076-82.

19 Fass R, Naliboff B, Higa L, et al. Differential effect of chronic esophageal acid exposure on mechano and chemesensitivity in humans. Gastroenterology 1998; 115: 1363-73.

20 Galmiche JP. Gastro-oesophageal reflux: does it matter what you eat? Gut 1998;42:318-19.

21 Meyer JH, Hlinka M, Tabrizi Y, et al. Inhibitions of sham and natural feeding in rats: Chemical specificities and intestinal distribution of feedback. Am f Physiol 1998;275: R1293-307.

22 Meyer JH, Hlinka M, Tabrizi Y, et al. Length of intestinal contact on nutrient driven satiety. Am F Physiol 1998;275: R1308-19.
23 Moran TH, Baldessarini AR, Salorio CF, et al. Vagal afferent and efferent contributions to the inhibition of food intake by cholecystokinin. Am F Physiol 1997;272:R1245-5

24 Zmarzty SA, Read NW. An examination of the effects of isoenergetic intragastric infusions of pure nutrients on cold pain perception in healthy human volunteers. Physiol Behav 1999;65:643-8

25 Melone J. Vagal receptors sensitive to lipids in the small intestine of the cat. F Auton Nerv Syst 1986;17:231-41.

26 Feinle C, Read NW. Ondansetron reduces nausea indused by gastroduodenal stimulation without changing gastric motility. Am f Physiol 1996;271:G591-7.

27 Feinle C, D'Mato M, Read NW. Cholecystohinin-A receptors mediate gastric sensory and motor responses to gastric distension and duodenal lipid Gastroenterology 1996;110:1379-85.

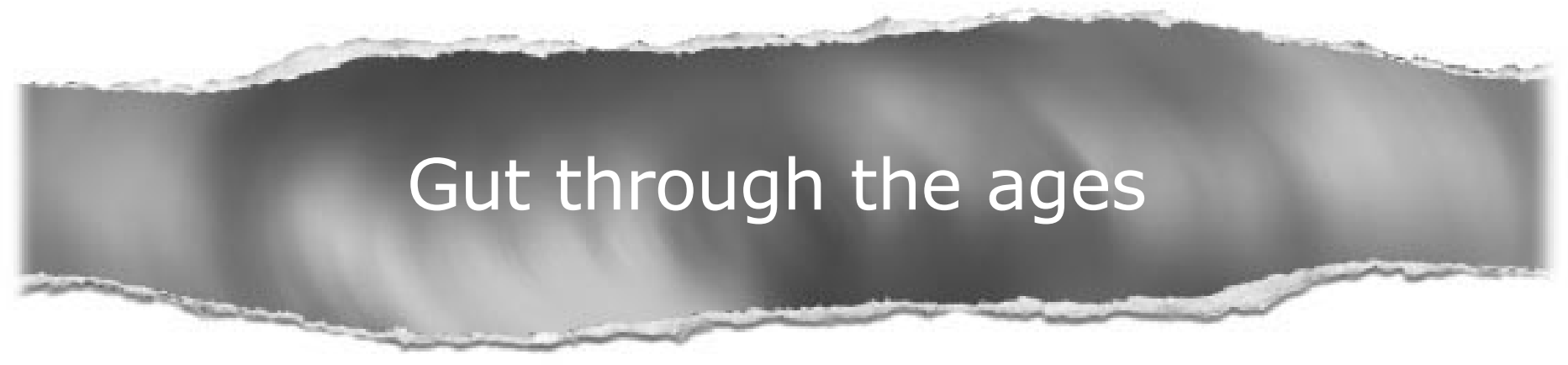

Browse the Archive

Gut online has an archive of content dating back to 1966 .

Full text from 1997; abstracts from 1975; table of contents from 1966

www.gutjnl.com 